Mathematical Modelling and Analysis

Volume 10 Number 4, 2005, PAGes 367-376

(c) 2005 Technika ISSN 1392-6292

\title{
THE NONLINEAR AND NONLOCAL INTEGRABLE SINE-GORDON EQUATION
}

\author{
P. MIŠKINIS \\ Department of Physics, Faculty of Fundamental Sciences, \\ Vilnius Gediminas Technical University
}

Sauletekio Ave 11, LT-10223, Vilnius-40, Lithuania

E-mail: paulius.miskinis@fm.vtu.lt

Received June 10, 2005; revised November 20, 2005

\begin{abstract}
A new type of the nonlocal sine-Gordon equation with the generalized interaction term is suggested. Its limit cases, symmetries and exact analytical solutions are obtained. This type of the nonlocal sine-Gordon equation is shown to possess one-, two- and $N$-solitonic solutions which are a nonlocal deformation of the corresponding classical solutions of the sine-Gordon equation.
\end{abstract}

Key words: nonlocality, SG equation, integrability

\section{Introduction}

The sine-Gordon equation (SGE)

$$
\phi_{t t}-a \phi_{x x}=b \sin (\lambda \phi)
$$

is one of the basic nonlinear equations both in mathematics and modern physics. In mathematics it appears as an equation for the surfaces of constant negative curvature $(a=\lambda=-b=1)$ and was known even to F. Minding and E. Beltrami. The physical applications are related with the description of dislocations in solid state physics [14], motion of Bloch magnetic walls in magnetic crystals [12], magnetic flux propagation in superconductors [8] and etc. [10]. In these applications the SGE gives the simplest nonlinear description of phenomena under consideration. More adequate models correspond to SGE generalizations (1.1).

All known nonlocal generalizations of SGE could be divided into two groups: 1) where the kinetic or 2) the dynamic term is under nonlocal generalization. Various generalizations where the local operator $\partial_{x x} \phi$ is replaced by the integro-differential operator $L[\phi]$ belong to the first group [1]:

$$
\phi_{t t}-L[\phi]=b \sin (\lambda \phi) .
$$


In particular, various interesting examples of nonlocal Josephson electrodynamics belong to the family of the evolution equation (1.2). These examples were introduced in [16]-[5], in which one of the basic model equations is

$$
\phi_{t t}-\hat{H} \phi_{x}+\sin \phi=0,
$$

where $\hat{H}$ is the Hilbert transform (see Appendix). The evolution equation (1.3) was an object of study in a series of papers $[4,6,15,16,17]$.

The nonlocal generalization of SGE proposed in [2] belongs to the first group

$$
\phi_{t t}-{ }^{R} D_{x}^{\alpha} \phi+\sin \phi=0,
$$

where ${ }^{R} D_{x}^{\alpha}$ is the Riesz partial fractional derivative (see Appendix). For this equation, a family of breather-like solutions (i.e. solutions that are localized in space and periodic in time) has been found numerically, and it has been shown that these entities are quite robust and can be generated in the course of evolution of initial states of a rather different shape.

Another type of nonlocal generalization of SGE was proposed in $[9,19]$ :

$$
\phi_{t t}-\phi_{x x}=-2 \cos \left[\frac{\phi(x, t)}{2}\right] \int f(x-y) \sin \left[\frac{\phi(y, t)}{2}\right] d y,
$$

where $f(x)=1 /\left(x^{4}+\sigma^{4}\right)$ or Gauss-type. It is shown that small amplitude solitons of the nonlocal SGE can create coupled states. The effect is due to a change of the dispersion which originated because of nonlocal nonlinearity. The evolution equation (1.5) in the general case could be generalized in the form

$$
\phi_{t t}-\phi_{x x}=F[\phi],
$$

where $F[\phi]$ is a function of $\phi(x, t)$.

In the current contribution, a new type of nonlocal SGE is suggested. Exact analytical solutions of this equation and its Lagrangian are considered.

\section{Nonlocal Generalization of Sine-Gordon Equation}

Let us consider a special type of the nonlocal SGE (NSGE):

$$
\phi_{t t}-a \phi_{x x}=b^{R} D_{x}^{-\alpha} \sin \left(\lambda^{R} D_{x}^{\alpha} \phi\right),
$$

where $a, b$ and $\lambda$ are constants and ${ }^{R} D_{x}^{\alpha}$ means a space fractional Riesz derivative of the order $\alpha$ (see Appendix). This equation belongs to the second group of the possible nonlocal generalizations of SGE (1.6), where the term of potential interaction is modified.

At first sight this equation looks very complicated, but actually it is an equivalent transformation of the interaction term. Indeed, in the case of linear dependence this term does not change.

In the case of small values of the parameter $\alpha$, the infinitesimal form of equation (2.1) is given as follows: 


$$
\phi_{t t}-a \phi_{x x}=b \sin \lambda \phi+\alpha L[\phi],
$$

where $L[\phi]$ is a local perturbation of the classical SGE, when at $\alpha \rightarrow 0$ the NSGE turns into the ordinary SGE (1.1).

In the case of small amplitudes $\left|\lambda^{R} D_{x}^{\alpha} \phi\right| \ll 1$, the NSGE turns into the linear Klein-Gordon equation with the "mass" term $\lambda b \phi$.

If $\phi(x, t)$ is a solution of the SGE, then the function

$$
\phi_{1}(x, t)=\frac{2 \pi n}{\lambda} \pm \phi\left(C_{1} \pm x, C_{2} \pm t\right), \quad n=0, \pm 1, \pm 2, \ldots,
$$

where $C_{1}, C_{2}$ are arbitrary constants, is also an exact solution of SGE. The signs in expression (2.3) could be chosen arbitrarily. Unfortunately, this does not hold for NSGE solutions, but would be useful for generating new solutions of NSGE by the known solution of SGE.

\subsection{The Lagrangian}

It could be verified that the NSGE (2.1) has the Lagrangian form:

$$
\left.L=\int_{-\infty}^{+\infty}\left\{\frac{1}{2}\left[\left({ }^{R} D_{x}^{\alpha} \phi_{t}\right)^{2}-\left({ }^{R} D_{x}^{1+\alpha} \phi\right)^{2}\right]+\frac{b}{\lambda}\left[1-\cos \left(\lambda^{R} D_{x}^{\alpha} \phi\right)\right)\right]\right\} d x .
$$

Thus, the equation of motion (2.1) could be derived by using the modified Noether theorem. For instance, the energy-momentum tensor $T_{i k}$ in the Minkowsky metric $\eta_{i k}$ :

$$
T_{i k}=\left({ }^{R} D_{x}^{\alpha} \phi_{i}\right)\left({ }^{R} D_{x}^{\alpha} \phi^{l}\right) g_{l k}-g_{i k} \mathcal{L},
$$

where $\mathcal{L}$ is the Lagrangian density in the expression (2.4).

\subsection{The travelling wave solution}

The NSGE has the travelling wave solution - a nonlocal generalization of one-solitonic solution.

a) Let $b \lambda\left(\mu^{2}-a k^{2}\right)>0$, then

$$
\phi(x, t)=\frac{4}{\lambda}{ }^{R} D_{x}^{-\alpha} \operatorname{arctg}\left\{\exp \left[ \pm \frac{b \lambda\left(k x+\mu t+\theta_{0}\right)}{\sqrt{b \lambda\left(\mu^{2}-a k^{2}\right)}}\right]\right\},
$$

where $k, \mu, \theta_{0}$ are arbitrary constants.

b) Let $b \lambda\left(\mu^{2}-a k^{2}\right)<0$, then

$$
\phi(x, t)=\frac{4}{\lambda}{ }^{R} D_{x}^{-\alpha} \operatorname{arctg}\left\{\exp \left[ \pm \frac{b \lambda\left(k x+\mu t+\theta_{0}\right)}{\sqrt{b \lambda\left(a k^{2}-\mu^{2}\right)}}\right]\right\},
$$

where $k, \mu, \theta_{0}$ like above are arbitrary constants. 


\subsection{The nonlocal generalization of the two-solitonic} Perring-Skirme solution

The travelling wave solutions (2.6) and (2.7) are particular cases of a more general type of solution. Let $f(x)$ and $g(t)$ be solutions of the ordinary differential equations

$$
\begin{aligned}
& \left(f_{x}^{\prime}\right)^{2}=A f^{4}+B f^{2}+C, \\
& \left(g_{t}^{\prime}\right)^{2}=-a C g^{4}+(a B+b \lambda) g^{2}-a A,
\end{aligned}
$$

where $A, B, C$ are arbitrary constants. Then the function

$$
\phi(x, t)=\frac{4}{\lambda}{ }^{R} D_{x}^{-\alpha} \operatorname{arctg}[f(x) g(t)]
$$

is a solution of the NSGE (2.1):

a) for $A=0, B=k^{2}>0, C>0, \mu^{2}=a k^{2}+b \lambda>0$, then

$$
\phi(x, t)=\frac{4}{\lambda}{ }^{R} D_{x}^{-\alpha} \operatorname{arctg}\left[\frac{\mu \operatorname{sh}\left(k x+A_{1}\right)}{k \sqrt{a} \operatorname{ch}\left(\mu t+B_{1}\right)}\right],
$$

where $k, A_{1}, B_{1}$ are arbitrary constants;

b) for $A=0, B=-k^{2}<0, C>0, \mu^{2}=b \lambda-a k^{2}>0$, then

$$
\phi(x, t)=\frac{4}{\lambda}{ }^{R} D_{x}^{-\alpha} \operatorname{arctg}\left[\frac{\mu \sin \left(k x+A_{1}\right)}{k \sqrt{a} \operatorname{ch}\left(\mu t+B_{1}\right)}\right]
$$

is the solution of NSGE, where $k, A_{1}, B_{1}$ are again arbitrary constants.

c) for $A=k^{2}>0, B=k^{2} \gamma^{2}>0, C=0, \mu^{2}=a k^{2} \gamma^{2}+b \lambda>0$, then

$$
\phi(x, t)=\frac{4}{\lambda}{ }^{R} D_{x}^{-\alpha} \operatorname{arctg}\left[\frac{\gamma}{\mu} \frac{e^{\mu\left(t+A_{1}\right)}+a k^{2} e^{-\mu\left(t+A_{1}\right)}}{e^{k \gamma\left(x+B_{1}\right)}+e^{-k \gamma\left(x+B_{1}\right)}}\right]
$$

is the solution of NSGE, where $k, A_{1}, B_{1}, \gamma$ are arbitrary constants.

\section{4. $N$-solitonic solutions}

For $a=1, b=-1$, and $\lambda=1$ in the NSGE, the $N$-solitonic solution has the form

$$
\begin{aligned}
& \phi(x, t)={ }^{R} D_{x}^{-\alpha} \arccos \left[1-2\left(\frac{\partial^{2}}{\partial x^{2}}-\frac{\partial^{2}}{\partial t^{2}}\right)(\ln F)\right], \\
& F=\operatorname{det}\left\|M_{i j}\right\|, \quad M_{i j}=\frac{2}{a_{i}+a_{j}} \operatorname{ch}\left(\frac{z_{i}+z_{j}}{2}\right), \\
& z_{i}= \pm \frac{x-\mu_{i} t+C_{i}}{\sqrt{1-\mu_{i}^{2}}}, \quad a_{i}= \pm \sqrt{\frac{1-\mu_{i}}{1+\mu_{i}}}
\end{aligned}
$$

where $\mu_{i}, C_{i}$ are arbitrary constants. 


\section{Integrability}

The classical SGE belongs to the family of the integrable evolutionary equations. Is it possible to prove the same statement for the NSGE?

Let us consider the $S O(2,1)$ linear integrable system:

$$
\Phi_{t}=U \Phi, \quad \Phi_{x}=V \Phi,
$$

where $U$ and $V$ take values in the Lie algebra $s o(2,1)$. This means that $U$ and $V$ may take the following two types:

$$
\begin{aligned}
\text { (i) }: U=\left(\begin{array}{ccc}
0 & C & B \\
-C & 0 & A \\
B & A & 0
\end{array}\right), & V=\left(\begin{array}{ccc}
0 & F & E \\
-F & 0 & D \\
E & D & 0
\end{array}\right), \\
(\text { ii }): U & =\left(\begin{array}{ccc}
0 & C & B \\
C & 0 & A \\
B & -A & 0
\end{array}\right), \quad V=\left(\begin{array}{ccc}
0 & F & E \\
F & 0 & D \\
E & -D & 0
\end{array}\right),
\end{aligned}
$$

where the coefficients $A, B, C, D, E$ and $F$ are suitable functions of $\phi$ and its (non)local derivatives.

For the case (i), the integrable condition for system (3.1) in the case of local functions and their derivatives is the Gauss equation of the imbedding of the pseudo-sphere $S^{1,1} \subset \mathbb{R}^{2,1}$, and for the case (ii) it is the Gauss equation of the imbedding of the hyperplane $H^{2} \subset \mathbb{R}^{2,1}$. Since SGE corresponds to the case of $H^{2} \subset \mathbb{R}^{2,1}$, let us consider the case (ii).

Let $l, m, n$ be an orthonormal frame of $\mathbb{R}^{2,1}$, and $-l^{2}=m^{2}=n^{2}=1$. The condition $l^{2}=-1$ is the equation for $H^{2} \subset \mathbb{R}^{2,1}$. As follows from the linear system (3.1), the integrability condition is

$$
U_{x}-V_{t}+[U, V]=0 .
$$

For the case (ii) it can be written in the following form:

$$
\left\{\begin{array}{l}
C_{x}-F_{t}+A E-B D=0 \\
B_{x}-E_{t}+C D-A F=0 \\
A_{x}-D_{t}+C E-B F=0 .
\end{array}\right.
$$

For given $B, C, E, F$ we can solve the two first equations of system (3.5)

$$
\begin{aligned}
& A=\frac{E_{t}-B_{x}}{C E-B F} B+\frac{F_{t}-C_{x}}{C E-B F} C, \\
& D=\frac{E_{t}-B_{x}}{C E-B F} E+\frac{F_{t}-C_{x}}{C E-B F} F,
\end{aligned}
$$

and insert them into the third equation:

$$
\begin{gathered}
\left(\frac{E_{t}-B_{x}}{C E-B F} B+\frac{F_{t}-C_{x}}{C E-B F} C\right)_{x}-\left(\frac{E_{t}-B_{x}}{C E-B F} E+\frac{F_{t}-C_{x}}{C E-B F} F\right)_{t} \\
+C E-B F=0 .
\end{gathered}
$$


The nonlinear partial differential equation which admits an $S O(2,1)$ linear integrable system $(C E-B F \neq 0)$ is (3.8). Moreover, equation (3.8) is the Gauss equation for $H^{2} \subset \mathbb{R}^{2,1}$, when $B, C, E, F$ are arbitrary functions of $\phi$ and its local derivatives. The main difference between the local and nonlocal cases is dependence of the coefficients $A, B, C, D, E$ and $F$ on the possible nonlocal derivatives. Let us consider a few examples.

Example 1. Let $B=F=0$, and the values of the coefficients $C$ and $E$ are $C=\sqrt{\lambda b} \cos \left(\lambda^{R} D_{x}^{\alpha} \phi / 2\right), E=\sqrt{\lambda b} \sin \left(\lambda^{R} D_{x}^{\alpha} \phi / 2\right)$. From equation (3.8) we get NSGE:

$$
\phi_{t t}-\phi_{x x}=-b^{R} D_{x}^{-\alpha} \sin \left(\lambda^{R} D_{x}^{\alpha} \phi\right) .
$$

Example 2. Let $B=F=0$, and the values of the coefficients $C$ and $E$ are $C=\cosh \left({ }^{R} D_{x}^{\alpha} \phi / 2\right), E=\sinh \left({ }^{R} D_{x}^{\alpha} \phi / 2\right)$, then we get nonlocal sinh-Laplace equation:

$$
\phi_{t t}+\phi_{x x}=-b^{R} D_{x}^{-\alpha} \sinh \left({ }^{R} D_{x}^{\alpha} \phi\right) .
$$

Example 3. Let $B=F=0, C=E=e^{D_{x}^{\alpha} \phi}$, then we have the nonlocal Liouville equation:

$$
\phi_{t t}+\phi_{x x}=-b^{R} D_{x}^{-\alpha} e^{\lambda^{R} D_{x}^{\alpha} \phi} .
$$

In the case $\alpha \rightarrow 0$, all the above considered nonlocal equations turn into the classical local form.

Thus, NSGE is an integrable nonlocal evolution equation, and the integrability approach allows us to generate new kinds of the nonlocal integrable sinh-Laplace and Liouville equations.

\section{Geometrical Approach}

The classical SGE describes the surface of a constant negative curvature imbedded in $D$-dimensional space. Regarding the Tschebyscheff coordinates, the first and second fundamental forms of the surface are

$$
\begin{aligned}
& \mathrm{I}=d s^{2}=\cos ^{2} \frac{\phi}{2} d t^{2}+\sin ^{2} \frac{\phi}{2} d x^{2}, \\
& \mathrm{II}=-d \vec{r} \cdot d \vec{n}=\cos \frac{\phi}{2} \sin \frac{\phi}{2}\left(d t^{2}-d x^{2}\right) .
\end{aligned}
$$

It is easy to verify that, the Gauss curvature $K$ of such surface is

$$
K=\frac{\operatorname{det} Q}{\operatorname{det} G}=\frac{b_{11} b_{22}-b_{12}^{2}}{g_{11} g_{22}-g_{12}^{2}}=-1,
$$

where $Q$ and $G$ are matrices of the second and first fundamental forms in expressions (4.1) and (4.2). The mean curvature $H=\operatorname{Sp}\left(G^{-1} Q\right)$ :

$$
H=\frac{g_{22} b_{11}-2 g_{12} b_{12}+g_{11} b_{22}}{g_{11} g_{22}-g_{12}^{2}}=-2 \operatorname{ctg} \phi .
$$


The quantities $K$ and $H$ in (4.3) and (4.4) express the geometrical contents of the SGE.

The classical way to derive the SGE is the substitution the Christoffel connection coefficients $\Gamma_{i j}^{k}$, which are determined by the coefficients of the first fundamental form $g_{i j}$,

$$
\Gamma_{i j}^{k}=\frac{1}{2} g^{k l}\left(\frac{\partial g^{i l}}{\partial \xi^{j}}+\frac{\partial g^{j l}}{\partial \xi^{i}}-\frac{\partial g^{i j}}{\partial \xi^{l}}\right)
$$

into the Gauss equation:

$$
\frac{\partial \Gamma_{i j}^{l}}{\partial \xi^{k}}-\frac{\partial \Gamma_{i k}^{l}}{\partial \xi^{j}}+\Gamma_{i j}^{s} \Gamma_{k s}^{l}-\Gamma_{i k}^{s} \Gamma_{j s}^{l}=b_{i j} b_{k}^{l}-b_{i k} b_{j}^{l},
$$

where $\xi^{i}=(t, x)$.

Here we can consider one simplification. In the case of the first fundamental form $d s^{2}=A^{2} d t^{2}+B^{2} d x^{2}$, the Gauss curvature could be obtained from the expression (see e.g. [11])

$$
K=-\frac{1}{A B}\left[\left(\frac{A_{t}}{B}\right)_{t}+\left(\frac{B_{x}}{A}\right)_{x}\right] .
$$

Indeed, the substitution of $A=\cos \phi / 2$ and $B=\sin \phi / 2$ into (4.7) leads to the classical SGE:

$$
\phi_{t t}-\phi_{x x}=K \sin \phi \text {. }
$$

In the case of the nonlocal value of the coefficients,

$$
A=\cos \left(D_{x}^{\alpha} \phi / 2\right), \quad B=\sin \left(D_{x}^{\alpha} \phi / 2\right),
$$

by substituting (4.9) into (4.7) we get the nonlocal generalization of the SGE:

$$
\phi_{t t}-\phi_{x x}=K D_{x}^{-\alpha} \sin \left(D_{x}^{\alpha} \phi\right) .
$$

Together with the coefficients of the second fundamental form,

$$
b_{11}=-b_{22}=\cos \left(D_{x}^{\alpha} \phi / 2\right) \sin \left(D_{x}^{\alpha} \phi / 2\right),
$$

according to equation (4.3), we can obtain the value of the Gauss curvature $K=-1$.

Thus, for the first and second fundamental forms,

$$
\begin{aligned}
& \mathrm{I}=\cos ^{2}\left(\frac{D_{x}^{\alpha} \phi}{2}\right) d t^{2}+\sin ^{2}\left(\frac{D_{x}^{\alpha} \phi}{2}\right) d x^{2}, \\
& \mathrm{II}=\cos \left(\frac{D_{x}^{\alpha} \phi}{2}\right) \sin \left(\frac{D_{x}^{\alpha} \phi}{2}\right)\left(d t^{2}-d x^{2}\right),
\end{aligned}
$$

the surface of a constant negative curvature $K=-1$ imbedded in $D$-dimensional space obeys the NSGE:

$$
\phi_{t t}-\phi_{x x}=D_{x}^{-\alpha} \sin \left(D_{x}^{\alpha} \phi\right) .
$$




\section{Conclusions}

The NSGE, like the ordinary SGE, has a Lagrangian form (2.4), one-(2.6)(2.7), two-(2.11)-(2.13) and $N$-solitonic (2.14) solutions. Despite the nonlocal nature of the interaction term in the evolution equation, this model possesses nonlocal deformations of localized solutions.

The asymptotic form has slowly falling tails $\phi(x) \sim x^{\alpha}$ which converge to zero at $\alpha<0$, as follows from explicit expressions of the solutions. At the same time the total value of the momenta $I[\phi]=\int_{-\infty}^{+\infty} \phi(x, 0) d x$ diverges for any $\alpha>-1$. This means a nonlocal distribution of the momenta, energy and related magnitudes.

From the asymptotic and infinitesimal form of NDGE (2.1) the corresponding dispersion relations,

$$
\omega^{2}-a k^{2}=\lambda b \quad \text { and } \quad \omega^{2}-a k^{2}=W(k),
$$

follow, where $W(k)$ corresponds to the Fourier transform for the linearized part of the $b \sin \lambda \phi+\alpha L[\phi]$ according to equation (2.2) and which are the Klein-Gordon and sine-Gordon modified dispersion relations.

The NSGE can be obtained from the discretized version of the evolution equation:

$$
y_{n}^{\prime \prime}=\frac{a}{s^{2}}\left(y_{n+1}-2 y_{n}+y_{n-1}\right)+\lambda y_{n} \cos \left[\lambda s^{-\alpha}\left(y_{n+1}-y_{n}\right)\right],
$$

where $s$ is the length of the space step displacement.

The variety of the physical origination of SGE (1.1) allows us to apply the obtained solutions not only to the Josephson effect $[3,5,7,15,16,17]$ but also to dislocations evolution in the modified Frenkel-Kontorova model [14] magnetic crystals [12], semiconductors [8] and so on.

Note here one important property. The continuous changes of the parameter $\alpha \in[0 ; 2]$ does not mean a continuous transition of one evolution equation to another. Let us have an evolution equation in the form

$$
\phi_{t t}-a \phi_{x x}=N_{\alpha}[\phi]
$$

where $N_{\alpha}[\phi]$ means the nonlocal operator on $\phi(x, t)$, and $\alpha$ is the parameter of nonlocality. The transformation of the operator $N_{\alpha}[\phi]$ for $\alpha \in[0 ; 2]$ induces the transformation of the automorphism groups $G_{0}$ and $G_{2}$ for the corresponding local evolution equations:

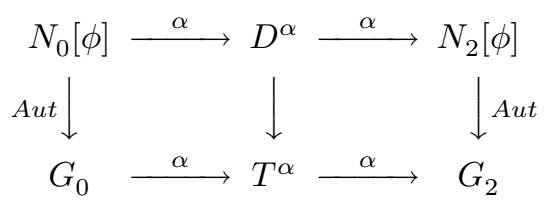

where, in the general case, the operator of the fractional derivative $D^{\alpha}$ induces an action on the group of translation operators $T^{\alpha}$. 


\section{Acknowledgements}

The author expresses his gratitude to Prof. L. Vázquez and Dr. T. Pierantozzi for useful discussions.

\section{Appendix}

To give an explicit expression for the Riesz pseudo-differential operator, we first introduce the Weyl fractional integrals $I_{ \pm}^{\beta}$ of the order $\beta>0[13,18]$ :

$$
I_{ \pm}^{\beta} \phi(x)=\left\{\begin{array}{l}
\frac{1}{\Gamma(\beta)} \int_{-\infty}^{x}(x-\xi)^{\beta-1} \phi(\xi) d \xi \\
\frac{1}{\Gamma(\beta)} \int_{x}^{+\infty}(\xi-x)^{\beta-1} \phi(\xi) d \xi
\end{array}\right.
$$

Then the Weyl fractional derivatives could be introduced by the relations

$$
D_{ \pm}^{\alpha} \phi(x)= \begin{cases} \pm\left(\frac{d}{d x} I_{ \pm}^{1-\alpha}\right) \phi(\xi), & 0<\alpha<1 \\ \left(\frac{d^{2}}{d x^{2}} I_{ \pm}^{2-\alpha}\right) \phi(x), & 1<\alpha<2\end{cases}
$$

where $I_{ \pm}^{\alpha}$ denotes the Weyl fractional integrals of the order $\alpha>0$. When $\alpha=0$, the Weyl fractional derivative degenerates into the identity operator

$$
D_{ \pm}^{0} \phi(x)=I \phi(x)=\phi(x) .
$$

For the continuity of $D_{ \pm}^{\alpha} \phi(x)$ with respect to $\alpha$,

$$
D_{ \pm}^{1}= \pm \frac{d}{d x}, \quad D_{ \pm}^{2}=\frac{d^{2}}{d x^{2}} .
$$

For arbitrary $\alpha$ we have the definition

$$
D_{ \pm}^{\alpha} \phi(x)=\left\{\begin{array}{l}
\frac{1}{\Gamma[\{\alpha\}]} \frac{d^{[\alpha]}}{d x^{[\alpha]}} \int_{-\infty}^{x} \frac{\phi(t) d t}{(x-t)^{1+\{\alpha\}}} \\
\frac{-1}{\Gamma[\{\alpha\}]} \frac{d^{[\alpha]}}{d x^{[\alpha]}} \int_{x}^{+\infty} \frac{\phi(t) d t}{(t-x)^{1+\{\alpha\}}}
\end{array}\right.
$$

where $\{\alpha\}$ and $[\alpha]$ are fractional and integer parts of the $\alpha>0$. The Riesz fractional derivative, denoted sometimes as $\partial^{\alpha} / \partial|x|^{\alpha}$, is defined as

$$
{ }^{R} D_{x}^{\alpha} \phi(x)= \begin{cases}-\frac{D_{+}^{\alpha}+D_{-}^{\alpha}}{2 \cos (\alpha \pi / 2)} \phi(x), & \alpha \neq 1, \\ \left(\frac{d}{d x} \hat{H}\right) \phi(x), & \alpha=1,\end{cases}
$$

where $\hat{H}$ is the Hilbert transformation

$$
\hat{H} \phi(x)=\text { v.p. } \frac{1}{\pi} \int_{-\infty}^{\infty} \frac{\phi(\xi)}{x-\xi} d \xi,
$$

and the integral is understood in the Cauchy principal value sense.

An important property of the Riesz fractional derivative ${ }^{R} D^{\alpha}$ is that it is a Fourier multiplier operator with the symbol $|k|^{\alpha}$. 


\section{References}

[1] G.L. Alfimov, V.M. Eleonsky and L.M. Lerman. Solitary wave solutions of nonlocal sine-Gordon equations. Chaos, 8, $257-271$.

[2] G.L. Alfimov, T. Pierantozzi and L. Vázquez. Numerical study of a fractional sine-Gordon equation. In: Fractional differentiation and its applications, FDA'04, Workshop preprints/proceedings, 644 - 649, 2004-1.

[3] G.L. Alfimov and I.D. Popkov. Magnetic vortices in a distributed Josephson junction with electrodes of finite thickness. Phys. Rev. B, 52(4503), 1995.

[4] G.L. Alfimov and V.P. Silin. Excitations in Abrikosov-Josephson small-scale vortex structures. J. Exp. Theor. Phys., 81(915), 1995.

[5] Yu.M. Aliev, K.N. Ovchinnikov, V.P. Silin and S.A. Uryupin. Perturbations of stationary solutions in a nonlocal model of a josephson junction. J. Exp. Theor. Phys., 80(551), 1995.

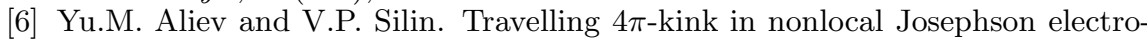
dynamics. Phys. Lett A, 177(259), 1993.

[7] Yu.M. Aliev, V.P. Silin and S.A. Uryupin. Theory of nonlinear dispersive waves in Josephson junctions, volume 5. 1992.

[8] A. Barone and G. Paterno. Physics and Applications of the Josephson Effect. Wiley, New York, 1982.

[9] M.D. Cunha, V.V. Konotop and L. Vázquez. Small-amplitude solitons in a nonlocal sine-Gordon model. Phys. Lett. A, 221(317), 1996.

[10] R.K. Dodd, J.C. Eilbeck, J.D. Gibbon and H.C. Morris. Solitons and Nonlinear Wave Equations. Academic Press, London, 1982.

[11] B. Dubrovin, S. Novikov and A. Fomenko. Contemporary geometry. Methods and applications. Nauka, Moscow, 1986.

[12] U. Enz. The motion of Bloch magnetic walls in magnetic crystals. Helv. Phys. Acta, 37(245), 1964.

[13] W. Feller. Meddelanden Lunds Universitets Matematiska Seminarium, volume 21. 1952.

[14] Ya.I. Frenkel and T.A. Kontorova. The model of dislocation in solid body. $Z h$. Eksp. Teor. Fiz., 8(1340), 1938.

[15] A. Gurevich. Nonlocal Josephson electrodynamics and pinning in superconductors. Phys. Rev. B., 46(3187), 1992.

[16] Yu.M. Ivanchenko and T.K. Soboleva. Nonlocal interaction in Josephson junctions. Phys. Lett., A 147, 65 - 69, 1990.

[17] R.G. Mintz and I.B. Snapiro. Dynamics of Josephson pancakes in layered superconductors. Phys. Rev. B, 49(6188), 1994.

[18] S.G. Samko, A.A. Kilbas and O.I. Marichev. Fractional Integrals and Derivatives. Theory and Applications. Gordon and Breach, Yverdon, 1993.

[19] L. Vázquez, W.A. Evans and G. Rickayzen. Numerical investigation of a nonlocal sine-Gordon model. Phys. Lett. A., 189(454), 1994.

\section{Netiesinè nelokali integruojamoji sine-Gordono lygtis}

\section{P. Miškinis}

Pasiūlyta nauja nelokali sine-Gordono evoliucinè lygtis su apibendrintu sąveikos nariu. Nustatyti šios lygties ribiniai atvejai, Lagranžianas, simetrijos, tikslūs analiziniai sprendiniai. Parodyta, kad šios rūšies nelokali sine-Gordono lygtis turi vieno, dvieju̧ bei $N$-solitoninius sprendinius, kurie yra atitinkamu̧ klasikiniu̧ sine-Gordono lygties sprendiniu̧ nelokalios deformacijos. Nelokalios sine-Gordono lygties integruojamumas siejamas su geometrinėmis dvimačiu̧ nelokaliai deformuotu̧ paviršiụ savybėmis. 